\title{
La rosa real: Una introducción a los números reales.
}

\author{
Miguel Delgado Pineda \\ Grupo de Innovación en Matemáticas. $\pi$-Mat \\ Departamento de Matemática Fundamentales, \\ Universidad Nacional De Educación a Distancia, UNED \\ C/ Senda del Rey 9 (28040 Madrid), \\ e-mail:miguel@mat.uned.es \\ Presentado en eXIDO17 (2017)
}

\section{RESUMEN}

En este trabajo se presenta una forma de presentar los números reales entre cero y uno de manera que facilita la adquisición del concepto de notación decimal tanto de números racionales como de números irracionales. En este trabajo se presenta una notación decimal binaria única para cada uno de estos números. Así se le facilita al estudiante la comprensión de las reglas aritméticas de números reales.

La presentación de la notación decimal binaria se deriva desde una interpretación geométrica inicial en relación a la medida de longitudes de segmentos, a la medida de áreas de rectángulos y a la medida de arcos o sectores de una circunferencia y círculo.

La razón primaria que nos movió ha realizar esta aportación didáctica se debe a que las tres formas usuales de introducir al conjunto de los números reales; utilizando cortaduras, mediante clases de equivalencia de sucesiones de números racionales y abordando la axiomática de la recta real, conlleva una seria dificultad didáctica para cualquier estudiante no universitario, como para los estudiantes de los primeros cursos universitarios para que estos comprendan la naturaleza del número real, la forma de notación decimal y las definiciones de la operaciones con estos números. A esta dificultad se le añade que el Calculo Matemático o Análisis Matemático que se imparte en esos primeros curso se fundamenta en el conjunto de números reales principalmente.

Palabras clave: Número real, Segmento lineal, Arco de curva, Notación binaria, Bisección de un segmento rectilíneo, Bisección de un rectángulo, Bisección de un arco de circunferencia.

\section{Introducción}

Una cuestión importante es que un profesor, antes de que se afronte una situación didáctica matemática en el aula, tenga conocimiento de la imagen visual que tienen los estudiantes de los números con los que se tratarán en esas situaciones didácticas.

Siempre que el autor, como profesor que inicia alguna sesión relativa a algunos conceptos de Cálculo o Análisis Matemático y sabiendo que los estudiantes son conscientes de la temática en la que se inician, ha solicitado a los estudiantes que pensaran un número. Una vez pensado, el profesor ha hecho un conjunto de supuesta adivinaciones sobre esos números que desconoce, pero de los cuales puede dar una serie de características. Casi siempre el estudiante ha pensado un número natural, con dos cifras como mucho. Rara vez es un número entero negativo y casi nunca el cero. En 
muy pocas ocasiones el número pensado es un número racional, y casi siempre ese número racional está descrito como fracción de números enteros. Suele ser muy raro que algún estudiante elija un número irracional, si bien los describen como una raíz cuadrada de un numero natural.

Esta práctica docente la puede experimentar el lector y así podrá estimar lo acertado de lo descrito, tanto si el lector es profesor de Enseñanza Universitaria como si es profesor de en Enseñanza Secundaria.

Con esta práctica queremos hacer emerger una gran dificultad didáctica en la cual se describe al número (real) como objeto para contar o como elemento para medir. Una pregunta interesante es saber si el estudiante, cuando trabaja con números, en general, tiene una imagen relativa a contar o tiene la imagen relativa a medir.

Cada conjunto numérico es familiar al estudiante con representaciones y reglas aritméticas y estructura de orden. Sin embargo, en Análisis Matemático es necesario disponer de las características topológicas de estos conjunto, sobre todo de los números reales.

Los números naturales, los más intuitivos para el estudiante, matemáticamente se justifican con los axiomas de Peano, y en ellos se establece por definición el orden del conjunto. Un buen orden donde cada subconjunto tiene un primer elemento.

El estudiante asume que con estos números él puede contar o relacionar conjuntos finitos con subconjuntos de números naturales. Sin embargo, no suele ser consciente de que este conjunto fundamenta el proceso de inducción que utilizaremos en este trabajo de forma recursiva.

Si bien la fundamentación de la definición de los números enteros no es excesivamente intuitiva, por el hecho de realizar clases de equivalencia de pares ordenados de números naturales. No es menos cierto que utilizar la notación usual de estos números permite extender el carácter intuitivo de los números naturales. De esta forma los números enteros positivos están identificados con los naturales y los números enteros negativos se presentan como el conjunto espejo de los naturales. No le resulta difícil asumir la operatoria entera como una extensión de la operatoria de los números naturales. Si bien el orden definido en el conjunto de los números enteros es un orden total, no es un buen orden. Sin embargo, este orden es un buen orden en cualquier subconjunto acotado inferiormente. Así pues, la imagen de estos números sigue manteniendo la idea de que sirven para contar, basta imaginar describir las plantas de un edificio para un ascensor. Es decir, los estudiantes cuentan tanto con números naturales como con números enteros.

La presentación de los racionales siempre ha presentado una cierta dificultad al estudiante neófito. Si bien la definición de número racional como clase de equivalencia del conjunto cociente del conjunto de pares de números enteros, resulta que la notación como fracción de enteros permite mantener la idea de que con los números racionales también se puede contar. Basta pensar en la posibilidad de contar las partes iguales de una tarta para entender la analogía mental con el proceso de contar, aunque la posibilidad de representar el mismo número con distintas fracciones de números enteros 
genera una cierta dificultad. Esto se aprecia en la identificación entre los números enteros y un subconjunto de números racionales por parte del estudiante.

El estudiante asume con cierta facilidad las operaciones con números racionales expresados como fracciones. Sin embargo, el orden total de dicho conjunto genera ciertas incertidumbres en el estudiante, puesto que este es un orden dividido. Es decir, dados dos números racionales, existe un número menor que uno y mayor que el otro, por ejemplo, basta pensar el número medio de los dos.

El orden de los números racionales nos permite afrontar la notación decimal de estos números. De hecho, cualquier número racional posee una expresión decimal finita o periódica. Ahora bien, la notación decimal no facilita imaginar todas las operaciones de números racionales, sobre todo con las expresiones periódicas.

En los números racionales se puede definir una distancia con facilidad empleando las operaciones aritméticas, y por tanto la forma de imaginarlos es mediante una línea recta, la línea recta racional.

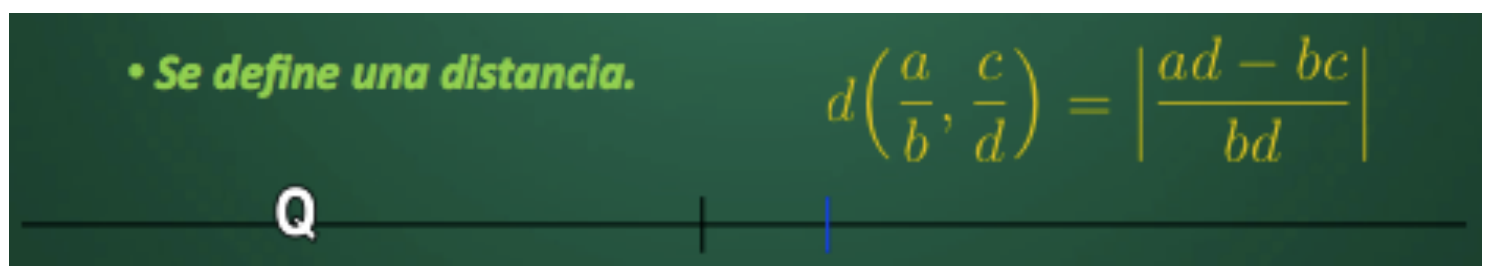

Figura 1: Representación de la recta racional.

El orden dividido permite bisecar cualquier intervalo racional. La bisección nos permite generar un proceso iterativos con los intervalos de números reales.

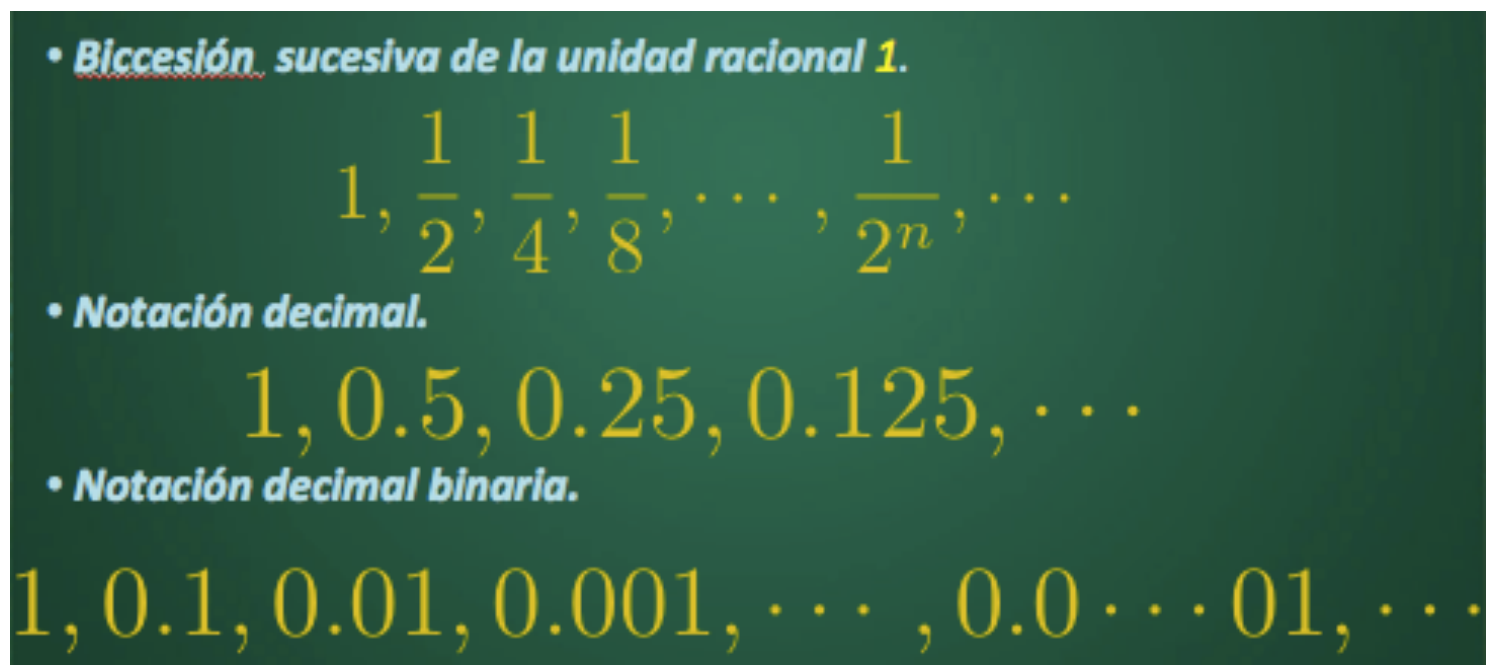

Figura 2: Sucesión al dividir sucesivamente la unidad.

En este trabajo la notación decimal binaria nos permite hacer una representación única para cada número real del intervalo $[0,1]$. 


\section{La bisección de objetos geométricos.}

La primera cuestión es disponer de un mecanismo para bisecar cualquier segmento rectilíneo del plano geométrico.

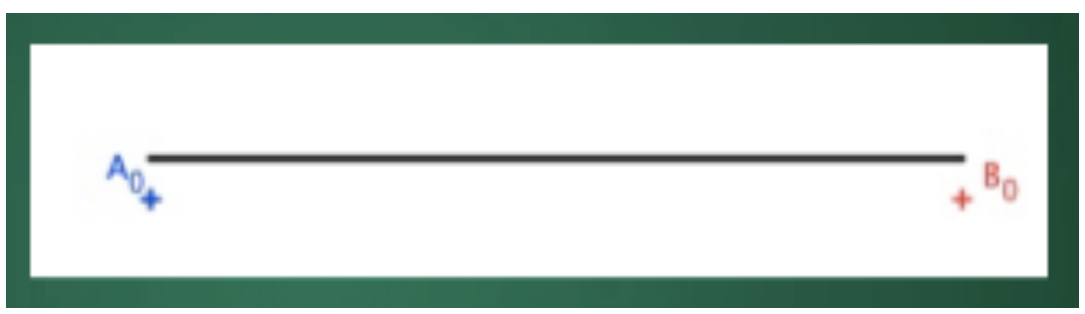

Figura 3: Segmento rectilíneo.

Sin duda, la determinación geométrica de la recta mediatriz a un segmento otorga una forma realizar esa división del segmento por el punto medio.

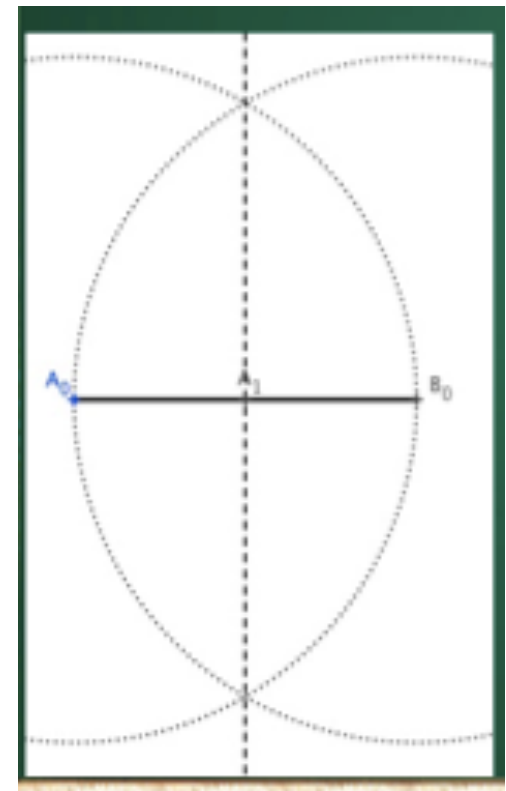

Figura 4: Determinación del punto medio.

Este proceso se puede repetir con uno de los dos subsegmentos, y así sucesivamente, obteniéndose un conjunto de puntos medios de cada segmento, y por tanto una secuencia de subsegmentos tal que la longitud de cada segmento de esta secuencia es la mitad de la longitud del segmento que le precede. Sin duda, la forma de bisecar subsegmentos genera diferentes sucesiones de subrectángulos. 


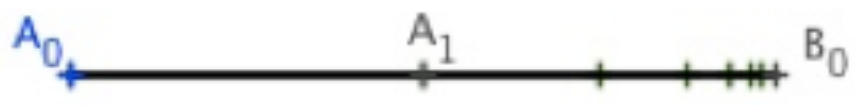

Figura 5: Secuencia de segmentos obtenidos pos subdivisión.

La segunda cuestión es disponer de un mecanismo para bisecar cualquier rectángulo del plano geométrico. En nuestro caso, podemos inicial de un rectángulo particular; un cuadrado.

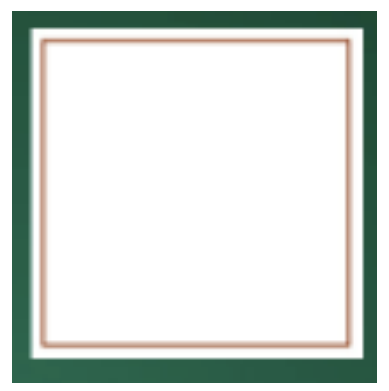

Figura 6: Rectángulo a subdividir.

Sin duda, la determinación del punto medio de uno de los lados del rectángulo y de la recta mediatriz a ese lado permite dividir el rectángulo inicial en dos subrectángulos.

Elegido uno de esos subrectánguos, como segundo paso se determina la mediatriz del lado que no fue dividido en el primer paso. De esta forma, al reiterar sucesivamente estos dos pasos, se obtiene sucesivamente los puntos medios de los lados de los rectángulos de forma alternativa en cada lado, y una secuencia de subrectángulos

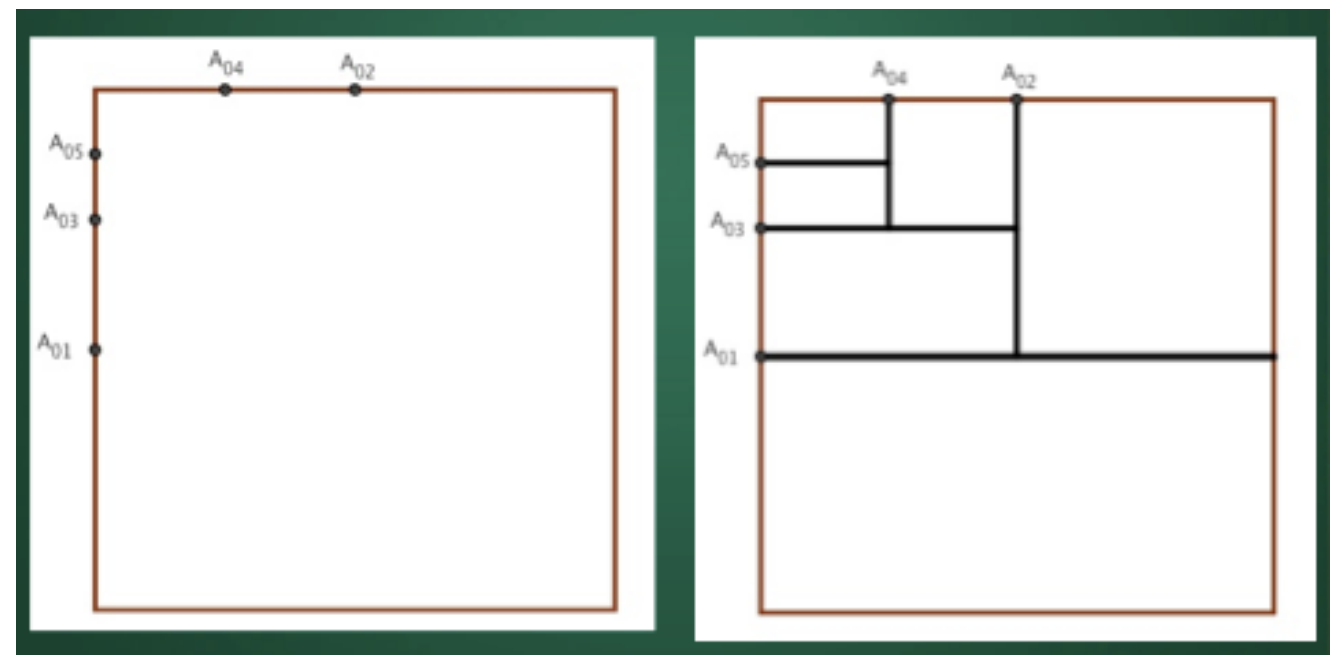

Figura 7: Determinación de una secuencia de subrectángulos.

El proceso iterativo genera una secuencia de subrectángulos tal que el área de cada rectángulo de esta secuencia es la mitad del área del rectángulo que le precede. Notemos 
que la forma de subdividir puede tener variaciones en generar la sucesión de subrectángulos.

La tercera cuestión es disponer de un mecanismo para bisecar cualquier sector circular, bisecando el arco circular del plano geométrico.

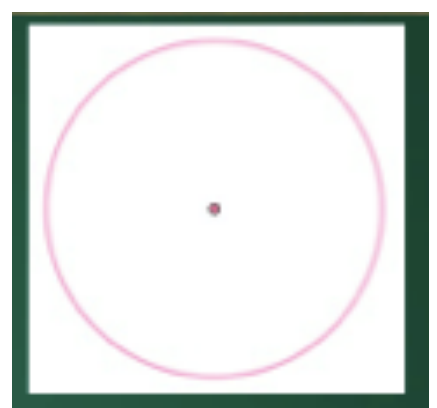

Figura 8: Circulo y circunferencia.

La determinación geométrica de la recta bisectriz a un ángulo otorga una forma realizar división de un sector circular mediante la determinación del punto medio del arco correspondiente.

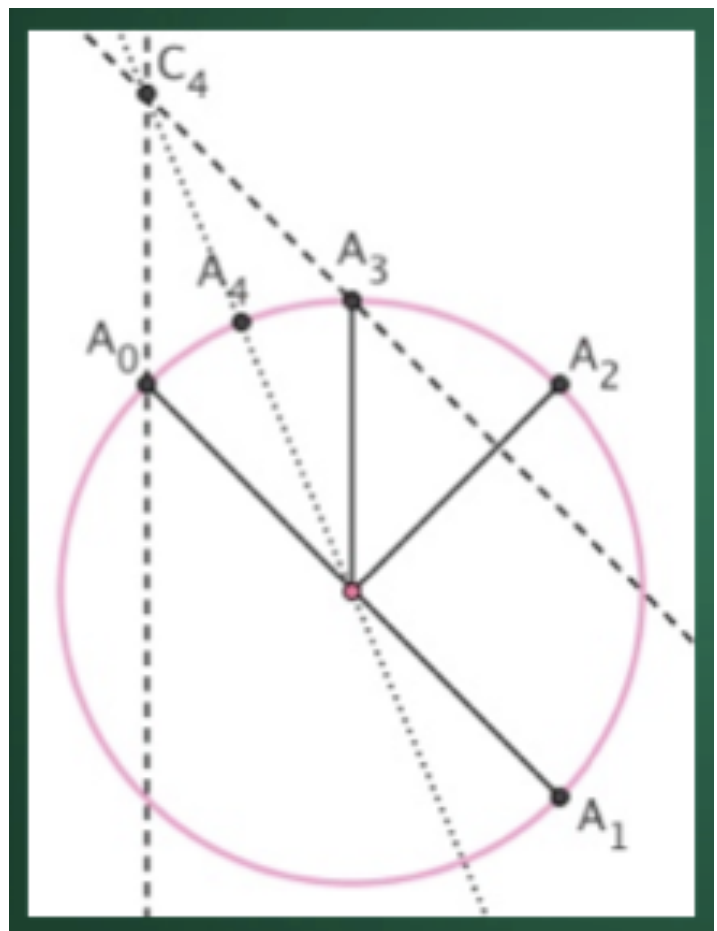

Figura 9: Determinación del punto medio de un arco circular.

Este proceso se puede repetir con uno de los dos subsegmentos, y así sucesivamente, obteniéndose un conjunto de puntos medios de cada arco circular, y por tanto una secuencia de tal que tal que la longitud del arco o el área del sector de cada sector de esta secuencia es la mitad de la longitud del arco o la mitad del área de sector que le precede. 


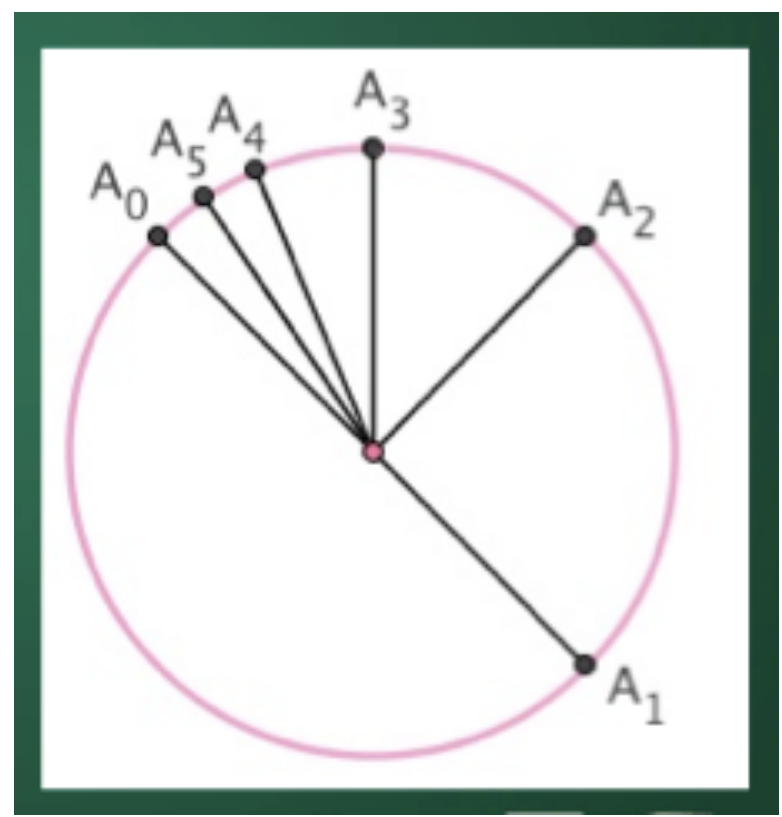

Figura 10: Secuencia de arcos y sectores circulares obtenidos por subdivisión.

Análogos procesos se podría generar para bisecar un paralelepípedo, o un sector esférico. En estos casos obtendríamos una secuencia de subparalelepípedos tales que el volumen de un paralelepípedo es la mitad del volumen del paralelepípedo que le precede.

Supuesto que la magnitud de cada una de las figura iniciales es 1, entonces las medidas (expresadas como número binario) de cada una de las piezas de la bisección sucesiva de cada figura de la sucesión son $0.1,0.01,0.001,0.0001,0.00001 \ldots$ respectivamente.

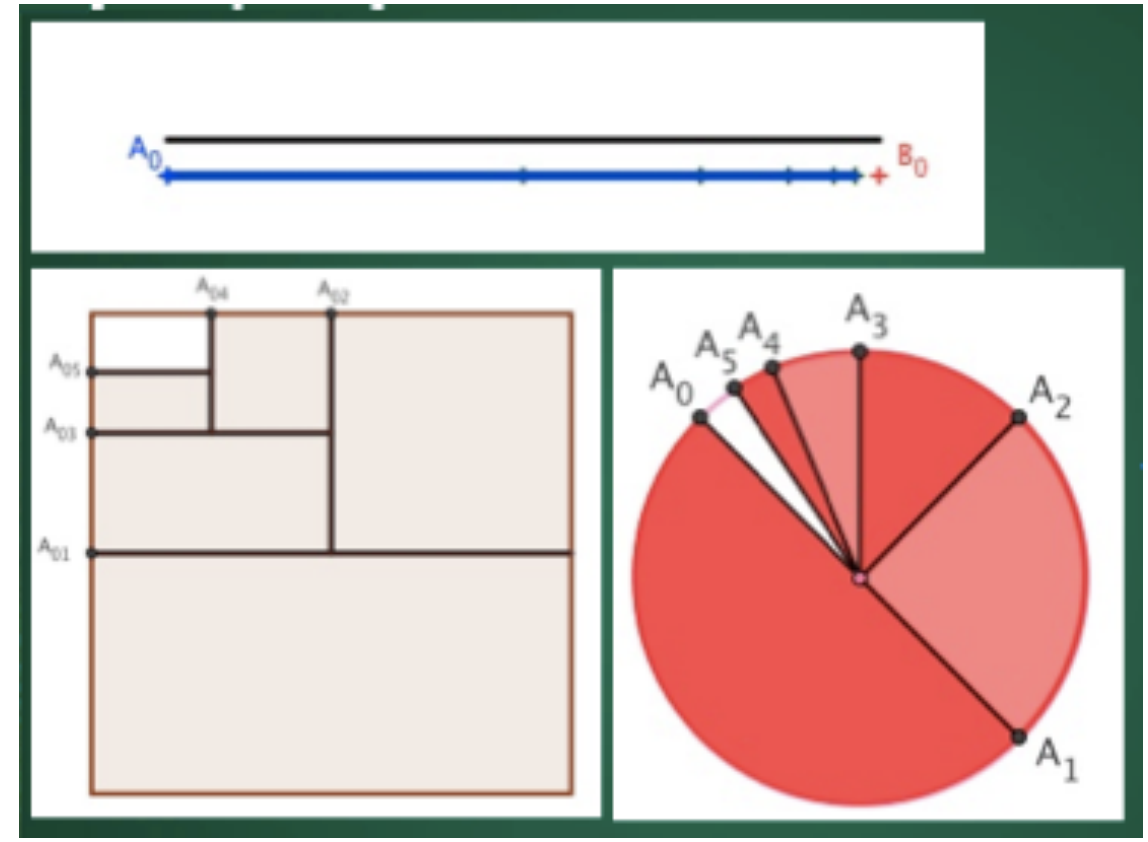

Figura 11: Primeros elementos de las secuencias de objetor obtenidos por bisección. 
La magnitud de la zona resaltada en la figura 11 es la suma de las magnitudes de las piezas resaltadas. Está es una operación elemental al disponer de la notación binaria pues basta poner el dígito binario 1 en la posición que indique la pieza. Por ejemplo,

$$
\begin{gathered}
0.1+0.01+0.001+0.0001+0.00001=0.11111 . \\
0.1+0.001+0.00001=0.10101 .
\end{gathered}
$$

\section{Los números racionales}

Algunos número racionales pueden ser expresados como una suma de algunos números de la secuencia, escrita en binario, $0.1,0.01,0.001,0.0001,0.00001 \ldots$ Sin embargo, no se pueden expresar todos los números racionales como suma de los elementos de la sucesión. Por ejemplo, un tercio no se puede obtener como suma de piezas. Baste recordar que la propiedad asociativa de la suma tan sólo nos permite realizar una suma de un conjunto finito de sumandos. Por tanto, debemos admitir escrituras decimales binarias no finitas al emplear un conjunto no finito de elementos de la sucesión. Por ejemplo,

- $1 / 3$ se escribe en notación binaria como $0.0101010101010101 \ldots$

- $1 / 9$ se escribe en notación binaria como $0.000111000111000111 \ldots$

- $5 / 9$ se escribe en notación binaria como $\quad 0.1000111000111000111 \ldots$

- $7 / 10$ se escribe en notación binaria como $0.1011001100110011 \ldots$

La situación que se presenta con este tipo de números y sus expresiones decimales, hace imprescindible establecer sentido a la "suma infinita" de elementos de la secuencia inicial. Es decir, la notación decimal "infinita" debe ser presentada al estudiante de forma que el proceso de aceptación tenga un carácter muy intuitivo y asuma expresiones escritas como la siguiente:

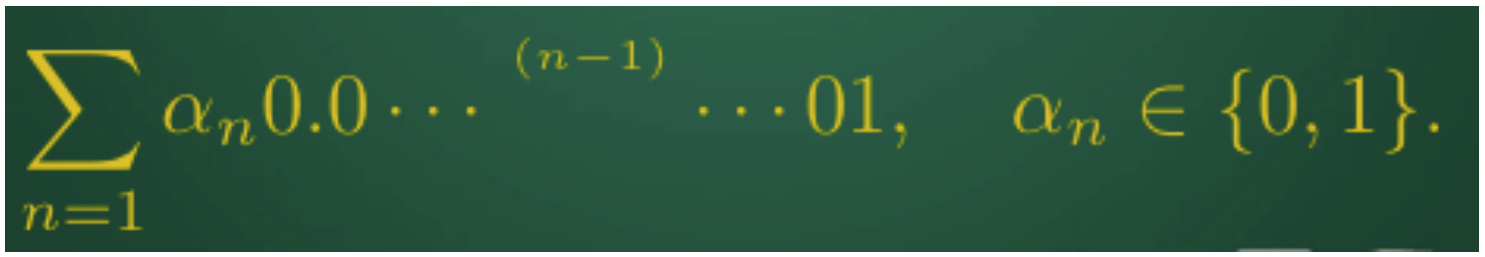

Figura 12: Expresión decimal infinita en binario.

Para poder asumir esta la notación decimal infinita nos apoyamos con las magnitudes de los segmentos, rectángulos y arcos. No es difícil dividir un segmento en tres subsegmentos de igual longitud, basta apoyarse en la utilización adecuada del teorema de Tales. Sin embargo, esta magnitud no puede ser descrita como la suma de un conjunto finito de subsegmentos.

Topamos de entrada con la paradoja de Aquiles y la tortuga pues por muchos segmentos que unamos describiendo el desplazamiento de Aquiles, resulta que la tortuga tiene un subsegmento de separación entre ella y Aquiles. 
Por otro lado, queremos dar sentido a la intuición, y si se estudia los movimientos de la tortuga y de Aquiles por separado, resulta que entendemos que la tortuga recorre una cierta distancia y Aquiles recorre esa distancia mas la que le separaba inicialmente de la tortuga. Desde este punto de vista se intuye que Aquiles y la tortuga se encuentran en un mismo punto de su camino rectilíneo.

Es muy intuitivo que la unión de cualquier conjunto de la sucesión de segmentos o de sectores circulares conforma un segmento o un sector circular. En particular, la unión de todos los elementos de la sucesión reconstruyen la figura inicial. Así pues, en términos de magnitud resulta que 1 puede ser escrito de forma binaria como $0.111111111 \ldots$ Es decir, para longitudes y áreas podemos definir la medida $\sigma$-aditiva.

$$
\sum_{n=1} \alpha_{n} 0.00 \ldots{ }^{(n-1)} \ldots 01 \text {, donde } \alpha_{n} \in\{0,1\}
$$

\section{- 1 se escribe como $0.111111111 .$.}

Figura 13: La magnitud unidad expresada en forma decimal binaria.

Ya se ha indicado que hay números racionales que tienen una expresión decimal binaria "infinita", y otros no. Sin embargo, apoyados en la expresión de la unidad como $0.111111111 \ldots$ nos permite escribir todos los números racionales con expresiones decimales no finitas. Por ejemplo, el número $1 / 8$ que es escrito en forma decimal finita como 0.001, puede ser escrito en la forma decimal no finita como $0.0001111111111 \ldots$

Con esta última consideración se tiene asegurada que cada número racional tiene una única expresión decimal binaria infinita. Ahora bien, esta expresión decimal es periódica, y tal que la expresión presenta agrupaciones de dígitos cero repetidos uniformemente y distribuidos en la expresión decimal binaria. Por ejemplo la expresión decimal binaria $0.010001000100010001 \ldots$ representa a un número racional. Otro es $0.1111001100110011 \ldots$

\section{Los números reales}

La analogía entre longitudes y números racionales avalan el modelo visual de los números racionales como se presenta en la figura 1 , al menos, en el intervalo de números racionales $[0,1]$. Es decir a cada número racional de ese intervalo le corresponde un segmento de una recta geométrica cuya longitud es ese número racional. Ahora bien, la longitud de cualquier segmento no puede ser descrito por un número racional, pues basta considerar una familia de subsegmentos de nuestra secuencia inicial para comprobar que no presentan regularidad de colocación de los dígitos cero. Es decir, nuestro modelo visual del conjunto de números racionales tiene huecos aunque no los podamos percibir por la vista.

De esta última reflexión emerge el número irracional con una expresión decimal binaria infinita donde los infinitos dígitos 0 no están uniformemente distribuidos en la 
expresión decimal binaria. Esto no permite dotar de magnitud a los segmentos rectilíneos que faltan. Por ejemplo, la expresión decimal binaria $0.101001000100001000001 \ldots$ se corresponde con un número irracional entre 0 y 1 . La sucesión de segmentos correspondientes son los de longitudes

$$
0.1,0.001,0.000001,0.000000001,0.00000000000001, \ldots \ldots
$$

La expresión $0.0101001010001010000101 \ldots$ es la expresión decimal binaria de otro número irracional.

Lo dicho con anterioridad no permite asumir que cualquier número real del intervalo de números reales $[0,1]$ tiene una expresión decimal infinita donde hay infinitos dígitos binarios 1 en la expresión. Salvo el número 0 para el cual todos sus dígitos binarios son 0 . Además, el estudio de la distribución de los dígitos 0 es esencial para saber si ese número real es racional o irracional. Se destaca que las expresiones decimales binarias con periodos son números racionales y el resto son números irracionales.

Si el existe sólo un número de dígitos 0 en la expresión, entonces el número es racional.

De esta forma los números reales quedan entendidos en relación a las magnitudes mencionadas, por ello que podamos entender la rosa de los números reales.

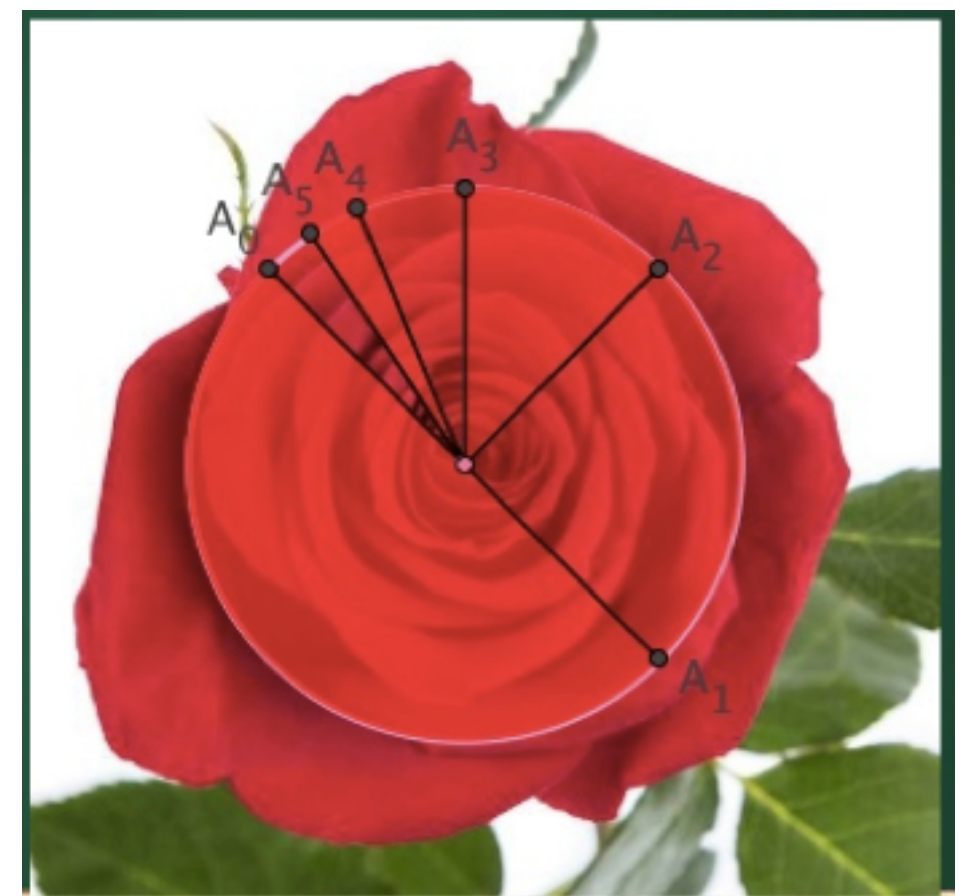

Figura 13: La magnitud unidad expresada en forma decimal binaria.

\section{Conclusiones}

Se ha establecido la analogía entre número y magnitud de forma intuitiva que permite presentar al estudiante de una forma sencilla sin necesidad de herramientas numéricas sofisticadas, pues sea empleado elementos y técnicas geométricas elementales. 
Se ha presentado una forma de expresar los números reales de forma única que hace uso de una notación decimal binaria infinita de forma que el dígito binario 1 se aparece en infinitas posiciones decimales.

Con lo dicho se puede atisbas que la expresión decimal infinita única facilita la forma de introducir las operaciones elementales de los números reales.

\section{Bibliografía}

M. Delgado Pineda \& M.J. Muñoz Bouzo. Lenguaje matemático, conjuntos y números. Ed Sanz-Torres. Madrid (2009) 